\title{
Assessment of Glycosuria and Bacterial Infections Amongst Prolonged - Healing Orthopaedic Wound Patients in Hospitals Within Imo State South Eastern Nigeria
}

\author{
Ifeanyi Odidika Clifford Obiajuru, ${ }^{1,}$, Chidinma Adanna Ikpeama ${ }^{2}$, \\ Immaculata Ogochukwu Uduchi ${ }^{3}$ \\ ${ }^{1}$ Department of Microbiology, Faculty of Medicine, Imo State University, Orlu Campus, Nigeria \\ ${ }^{2}$ Department of Animal and Environmental Biology, Imo State University, Owerri, Nigeria \\ ${ }^{3}$ Department of Microbiology \& Parasitology, Imo State University Teaching Hospital, Orlu, Nigeria
}

Email address:

drifeanyi_oc@yahoo.co.uk (I. O.C. Obiajuru),chidinmaikpeama2015@gmail.com (C. A. Ikpeama),ogoouduchi@gmail.com (I. O. Uduchi) ${ }^{*}$ Corresponding author

\section{To cite this article:}

Ifeanyi Odidika Clifford Obiajuru, Chidinma Adanna Ikpeama, Immaculata Ogochukwu Uduchi. Assessment of Glycosuria and Bacterial Infections Amongst Prolonged - Healing Orthopaedic Wound Patients in Hospitals Within Imo State South Eastern Nigeria. American Journal of Laboratory Medicine. Vol. 2, No. 3, 2017, pp. 34-40. doi: 10.11648/j.ajlm.20170203.12

Received: MM DD, 2017; Accepted: MM DD, 2017; Published: August 18, 2017

\begin{abstract}
Glycosuria and bacterial infections of patients with prolonged orthopaedic wounds and the antibiotics susceptibility of the bacteria associates was studied between October 2016 and May 2017. A total of 130 male and female respondents aged 18 to 50 years were selected for this study from out - patients and in - patients at Imo State University Teaching Hospital Orlu, Federal Medical Centre Owerri and 4 major private hospitals in Orlu and Owerri. One hundred and twenty four $(82.7 \%)$ respondents completed and returned their questionnaires and wound swab was collected from 101 $(67.3 \%)$ respondents. Thirteen $(12.9 \%)$ respondents had concomitant infections of 2 bacterial species each and $88(87.1 \%)$ had single bacterial infection each. Five species of bacteria: Staphylococcus aureus, Streptococcus species, Pseudomonas aeruginosa, Proteus mirabilis and Escherichia coli were isolated from the patients. A total of 114 bacterial isolates were obtained. The most prevalent isolate $(32.7 \%)$ was Pseudomonas aeroginosa and the least prevalent bacteria (12.8\%) was Streptococcus species. Out of 124 respondents that completed and returned their questionnaires, 66 (53.2\%) had wounds caused by vehicle road accident, 40 (32.3\%) had wounds caused by assault / domestic violence and 16 (12.9\%) had wounds caused by burn. The antibiotic susceptibility of the test isolates showed that ciprofloxacin (ciproxin) exhibited the highest growth inhibitory effect on Pseudomonas aeruginosa, Staphylococcus aureus, Streptococcus species, Proteus mirabilis and Escherichia coli. Nitrofurantoin did not exhibit any growth inhibitory effect on the test isolates except one strain of Escherichia coli. Ampiclox and Augmentin did not exhibit any growth inhibitory effect on Streptococcus species. Augmentin exhibited the lowest growth inhibitory effect on Staphylococcus aureus, Proteus mirabilis and Pseudomonas aeruginosa while Nitrofurantoin exhibited the lowest on Escherichia coli. Urine analysis of the patients showed that 54 (53.5\%) out of 101 patients examined had glucose in their urine. Comparative analysis of the data showed that prevalence of long - healing orthopaedic wounds was higher amongst patients with glucose in their urine than those without glucose in their urine. This study has shown that microbial infections which complicate and contribute immensely to long time taken for orthopaedic wounds to heal are treatable with conventional antibiotics available in Nigerian markets and used in Nigerian hospitals.
\end{abstract}

Keywords: Prolonged - Healing, Wounds, Out - Patients, In - Patients, Imo State Nigeria

\section{Introduction}

Orthopaedic wound is a wound that occurs in response to hard and/or soft tissue trauma. This includes injuries to bone, muscle, ligament and tendons, as well as spinal cord injuries. All forms of orthopedic trauma put patients at increased risk 
for infection. Open fractures (fractures in which the skin is broken) put patients at higher risk for infection. Wound and bone infections can be disabling for patients because they can delay or prevent healing. Previous studies [1] reported that wound healing in orthopaedic care is affected by the causes of the wound, as well as concomitant therapies used to repair musculoskeletal structures. Other workers $[2,3]$ reported that in wounds where oxygenation is not restored, healing is impaired. Temporary hypoxia after injury triggers wound healing, but prolonged or chronic hypoxia delays wound healing. Promoting the health of the host and creating an environment to foster natural healing processes is essential for helping to restore skin integrity. Accurate wound assessment and knowledge of nursing implications with specific wound care measures (cleansing, debridement, and dressings) is important for quality care.

Micro-organisms enter the body through breaks in the skin especially puncture wounds and other injuries causing infections that are difficult to treat. Disease -carrying bacteria, viruses, and parasites that get into the body can destroy healthy tissue, multiply and spread through blood. Infection of skin and other soft tissue can lead to infection of bones (osteomyelitis) and joints (septic arthritis). Without prompt treatment, orthopaedic infections can become chronic. Thus, even a scratch on the fingertip has the potential to permanently disable a patient's hand. Infections of the joint sometimes develop from an internal hip or knee replacement device (prosthesis). Infection occurs through direct contact with an infected person or through indirect contact like contaminated object. Some infections like orthopaedic wounds are difficult to treat [4]. Depending upon severity and other factors, osteomyelitis can cause irreversible damage (necrosis) to bone cells. Doctors often prescribe antibiotics and may need to drain the infection and/or cut away (debride) dead bone and other infected tissue. Difficult cases can require amputation. Early diagnosis, appropriate antibiotic therapy, and surgical intervention when required may be necessary to cure most infections and prevent permanent problems. Antibiotics successfully treat most infections caused by bacteria. However, some microorganisms develop resistance to standard antibiotic treatments. When using an antibiotic, bacteria resistant to treatment may survive and multiply. These bacteria can create infections for which there is no treatment. Resistant bacteria may spread to other people, posing a major health threat for everybody. It is therefore important to follow the directions of the doctor and complete the prescribed dosage, even if the patient feels well after taking a few dose.

Certain chronic diseases like HIV, rheumatoid arthritis, diabetes mellitus, hemophilia, and sickle cell anaemia put a patient at greater risk for infections. In the management of infections, it is valuable to investigate the existence and interference of these diseases to avoid treatment failure or delays. Simple laboratory investigations like urine analysis and rapid tests for HIV and rheumatoid factor may reveal a lot. Normal urine does not contain glucose because the kidneys are able to reabsorb all of the filtered glucose from the tubular fluid back into the bloodstream. When glucose is excreted into the urine, it is referred to as glycosuria. Glycosuria is nearly always caused by elevated blood glucose levels, most commonly due to untreated diabetes mellitus [5]. Rarely, it may be caused by an intrinsic problem with glucose re-absorption within the kidneys (such as Fanconi syndrome), producing a condition known as renal glycosuria [6]. Glycosuria leads to excessive water loss into the urine with resultant dehydration, a process called osmotic diuresis.

\section{Materials and Methods}

\subsection{Materials}

\subsubsection{Study Area}

This study was carried out at 2 tertiary health institutions in Imo State: Federal Medical Centre Owerri and Imo State University Teaching Hospital, Orlu. Imo State situates in Southeastern Nigeria and lies between latitudes $5^{\circ} 30^{\prime}$ and $6^{\circ}$ $15^{\prime}$ North, longitude $6^{\circ} 38^{\prime}$ and $7^{\circ} 18^{\prime}$ East. It is densely populated with men and women of all ages engaged in different walks of life. Orthopaedic wound is one of the most common cases in hospitals within the State and Nigeria in general. Most cases leave patients admitted for several months and even years.

\subsubsection{Study Samples}

The samples used for this study are wound swabs and early morning urine collected from men and women with orthopaedic wounds in hospitals within Imo State, South eastern Nigeria.

\subsubsection{Test Antibiotics}

The antibiotic discs selected for this study are commercially prepared discs of conventional antibiotics commonly used in Imo State for treatment of common infections namely: Zinnat (cefuroxime), Azycin (Azithromycin), Augmentin (Amoxycillin/Clavulanate potassium), Avicef (Ceftriaxone), Sparflox, Levitol (Levofloxacin), Gaxin (Ofloxacin), Ciproxin, Streptomycin, Ampiclox, Gentamycin and Nitrofurantoin.

\subsubsection{Instrument for Data Collection}

The instrument used for collection of data in this study is a structured questionnaire comprising 3 sections: Section A: Socio - demographic data and Section B: Clinical Records.

\subsection{Methods}

\subsubsection{Selection of Respondents}

A total of 130 male and female respondents aged 18 to 50 years were selected for this study. The respondents were selected from orthopaedic wound patients receiving care at various hospitals within Imo State.

Nurses in the accident and emergency and orthopaedic wards of the hospitals were recruited as field assistants for administration of questionnaires and collection of samples 
for laboratory examination. The patients were approached individually through the field assistants and the objectives of the study explained to them. Their willingness to participate in the study was requested and those who indicated willingness were enlisted and given questionnaires to complete and return. Those who could not write were assisted by the field assistants to fill the questionnaires.

\subsubsection{Method of Sample Collection}

Sterile Evepon swab sticks were used to collect wound swab from each patient in the morning hours prior to dressing of the wound. Sterile disposable urine bottles were used to collect early morning urine samples. The samples were labeled with the respondent's index number indicated on the research questionnaire, and transported to the Microbiology Laboratory Imo State University Teaching Hospital Orlu for analysis within 30 minutes.

\subsubsection{Bacteriological Analysis of Samples}

The wound swabs collected from the patients were analyzed by culture technique using chocolate and MacConkey agar plate as in [7]. The plates were examined for microbial growth and different isolates obtained were identified using standard methods as described by [7, 8]. The findings were compared with Bergy's manual of determinative bacteriology [9].

\subsubsection{Antimicrobial Effects of Test Isolates}

The microbial isolates from wound swabs of patients were tested for antibiotic susceptibility using commercially prepared antibiotic discs as described by [7].

\subsubsection{Examination of Urine Samples}

All urine samples collected from the patients were examined for glucose using rapid test strips (Meditest ${ }^{\circledR}$ Combi 9).

\subsection{Method of Data Analysis}

The data obtained from this study were analyzed statistically using Chi square and Analysis Variance (ANOVA) as in [10].

\section{Results}

Out of 130 patients selected for this study, 124 (82.7\%) patients completed and returned their questionnaires, 101 $(67.3 \%)$ patients gave consent for wound swab to be collected from their wounds. Of this number, 13 (12.9\%) had concomitant infections of 2 bacterial species each and 88 $(87.1 \%)$ had single bacterial infection each. Generally 5 species of bacteria: Staphylococcus aureus, Streptococcus species, Pseudomonas aeruginosa, Proteus mirabilis and Escherichia coli were isolated from the patients. A total of 114 bacterial isolates were obtained. The most prevalent isolate $(32.7 \%)$ was Pseudomonas aeroginosa and the least prevalent bacteria (12.8\%) was Streptococcus species.

\subsection{Prevalence of Bacteria Infections Amongst Orthopaedic Wound Patients}

Out of 101 patients who participated in the study, 19 were aged $18-25$ years, 44 were aged $26-35$ years, 29 were aged 36 - 45 years and 9 were aged 46 years and above. Amongst the age group 18 - 25 years, 5 (41.7\%) out of 12 males were infected with Staphylococcus aureus, 3 (25\%) were infected with Escherichia coli, 2 (16.7\%) were infected with Pseudomonas aeruginosa, 2 (16.7\%) were infected with Proteus mirabilis, and $2(16.7 \%)$ were infected with Streptococcus species. Seven females were examined, 3 (42.9\%) were infected with Proteus mirabilis, 1 (14.3\%) had Streptococcus species infection, 1 (14.3\%) was infected with Staphylococcus aureus, 1 (14.3\%) was infected with Escherichia coli and 1 (14.3\%) was infected with Pseudomonas aeruginosa. Amongst the age group $26-35$ years, 30 males and 14 females were examined. Of this number, 7 (23.3\%) males and 7 (50\%) females were infected with Staphylococcus aureus, 7 (23.3\%) males and 6 (42.9\%) females were infected with Pseudomonas aeruginosa, 7 $(23.3 \%)$ males and 1 (7.1\%) female had Proteus mirabilis infection, 6 (20\%) males and $2(14.3 \%)$ females had Streptococcus species infection and $4(13.3 \%)$ males and 2 (14.3\%) females had Escherichia coli infection. Amongst the age group 36 - 45 years, 22 male and 7 females were examined. Seven $(31.8 \%)$ males and $2(28.6 \%)$ females had Staphylococcus aureus infection, $6(27.3 \%)$ males and 2 (28.6\%) females had Escherichia coli infection, 11 (50\%) males and $1(14.3 \%)$ female had Pseudomonas aeruginosa infection, 2 (9.1\%) males and 1 (14.3\%) female had Proteus mirabilis infection and $1(4.6 \%)$ male and $1(14.3 \%)$ female had Streptococcus species infection. Amongst the age group 46 years and above, 8 male and 1 female were examined. The only female in this group had Proteus mirabilis infection, while $3(37.5 \%)$ males had Staphylococcus aureus infection, $3(37.5 \%)$ had Pseudomonas aeruginosa infection, 2 (25\%) had Escherichia coli infection, and 1 (12.5\%) had Proteus mirabilis infection. Table 1 summarizes age and gender related prevalence of infection amongst patients with orthopaedic wounds. As shown, the highest prevalence of infection (50\%) was observed amongst males aged $36-45$ years and females aged $26-35$ years. Statistical analysis of the data using Chi square showed no significant difference $(p>0.05)$ between age and / gender and prevalence of infection.

Table 2 summarizes duration of wound - related prevalence of infection. As shown, out of 3 patients with 1 to 3 months old wounds, 2 (66.7\%) were infected with Proteus mirabilis while 1 (33.3\%) was infected with Staphylococcus aureus. Seventeen patients had 4 to 6 months old wounds, 6 (35.3\%) were infected with Pseudomonas aeruginosa, 5 (29.4\%) patients were infected with Escherichia coli, 4 $(23.5 \%)$ were infected with Staphylococcus aureus, 3 (17.6\%) were infected with Proteus mirabilis and 2 (11.8\%) were infected with Streptococcus species. Thirty patients had 7 to 9 months old wounds, $11(36.7 \%)$ were infected with 
Pseudomonas aeruginosa, $8(26.7 \%)$ were infected with Staphylococcus aureus, 5 (16.7\%) were infected with Streptococcus species, $4(13.3 \%)$ were infected with Escherichia coli and 4 (13.3\%) were infected with Proteus mirabilis. Fifty patients had 10 to 12 months old wounds, 18 (36\%) were infected with Staphylococcus aureus, 16 (32\%) were infected with Pseudomonas aeruginosa, 11 (22\%) were infected with Escherichia coli, 7 (14\%) were infected with Proteus mirabilis and 6 (12\%) were infected with Streptococcus species. One patient (5.9\%) had more than 12 months old wound and was not infected with bacterial.

\subsection{Antibiotic Susceptibility Test}

Table 3 summarized the antibiotic susceptibility of the test isolates. As shown, ciprofloxacin (ciproxin) exhibited the highest growth inhibitory effect on Pseudomonas aeruginosa, Staphylococcus aureus, Streptococcus species, Proteus mirabilis and Escherichia coli. Nitrofurantoin did not exhibit any growth inhibitory effect on the test isolates except one strain of Escherichia coli. Ampiclox and Augmentin did not exhibit any growth inhibitory effect on Streptococcus species. Augmentin exhibited the lowest growth inhibitory effect on Staphylococcus aureus, Proteus mirabilis and Pseudomonas aeruginosa while Nitrofurantoin exhibited the lowest on Escherichia coli.

\subsection{Relationship Between Glycosuria and Duration of Orthopaedic Wounds}

Table 4 summarizes the prevalence of glycosuria amongst patients with prolonged healing orthopaedic wounds. As shown, out of 3 patients with 1 to 3 months old orthopaedic wounds, none had glucose in the urine. Out of 17 patients with 4 to 6 months old orthopaedic wounds, 5 (2.9\%) had glucose in their urine. Out of 30 patients with 7 to 9 months old orthopaedic wounds, $17(56.7 \%)$ had glucose in their urine. Out of 50 patients with 10 to 12 months old orthopaedic wounds, $31(62 \%)$ had glucose in their urine. One patient with more than 12 months old orthopaedic wound had glucose in his urine. Generally, 54 (53.5\%) out of 101 patients examined had glycosuria. Comparative analysis of the data (Figure 1) showed that prevalence of prolonged healing orthopaedic wounds was higher amongst patients with glycosuria than those without glucose in their urine. Analysis of the data using ANOVA showed strong positive correlation $(\mathrm{p}<0.05)$ between glycosuria and prolonged healing orthopaedic wounds.

Table 1. Age and Gender - Related Prevalence of Infection.

\begin{tabular}{|c|c|c|c|c|c|c|c|}
\hline \multirow{2}{*}{ Age (years) } & \multirow{2}{*}{ Sex } & \multirow{2}{*}{ No Exam } & \multicolumn{5}{|c|}{ Number Infected (\%) } \\
\hline & & & Staph aureus & Esch. coli & Pseudomonas aeruginosa & Proteus mirabilis & Strept species \\
\hline \multirow{2}{*}{$18-25$} & Male & 12 & 5 & 3 & 2 & 2 & 2 \\
\hline & Female & 7 & 1 & 1 & 3 & 1 & 1 \\
\hline \multirow{2}{*}{$26-35$} & Male & 30 & 7 & 3 & 7 & 7 & 6 \\
\hline & Female & 14 & 7 & 2 & 6 & 1 & 2 \\
\hline $36-45$ & Male & 22 & 7 & 6 & 11 & 2 & 1 \\
\hline \multirow{2}{*}{46 - above } & Male & 8 & 3 & 2 & 3 & 1 & 0 \\
\hline & Female & 1 & 0 & 0 & 0 & 1 & 0 \\
\hline Total & & 101 & $32(31.7)$ & $20(19.8 \%)$ & $33(32.7 \%)$ & $16(15.8 \%)$ & $13(12.8 \%)$ \\
\hline
\end{tabular}

Table 2. Relationship between duration of Wound and Prevalence of Microbial Infections.

\begin{tabular}{|c|c|c|c|c|c|c|}
\hline \multirow{2}{*}{$\begin{array}{l}\text { Duration of } \\
\text { wound (Months) }\end{array}$} & \multirow{2}{*}{ Number Exam } & \multicolumn{5}{|c|}{ Number Infected (\%) } \\
\hline & & Staph. aureus & Esch. coli & Pseudomonas aeruginosa & Streptococ species & Proteus mirabilis \\
\hline $1-3$ & 3 & 1 & 0 & 0 & 0 & 2 \\
\hline $4-6$ & 17 & 4 & 5 & 6 & 2 & 3 \\
\hline $7-9$ & 30 & 8 & 4 & 11 & 5 & 4 \\
\hline $10-12$ & 50 & 18 & 11 & 16 & 6 & 7 \\
\hline$>12$ & 1 & 1 & 0 & 0 & 0 & 0 \\
\hline Total & 101 & $32(31.7 \%)$ & $20(19.8 \%)$ & $33(32.7 \%)$ & $13(12.8 \%)$ & $16(15.8 \%)$ \\
\hline
\end{tabular}

Table 3. Antibiotic Susceptibility Pattern of Test Bacterial Isolates.

\begin{tabular}{|c|c|c|c|c|c|c|c|c|c|c|c|c|c|}
\hline \multirow{2}{*}{ Isolate } & \multirow{2}{*}{ No Exam } & \multicolumn{12}{|c|}{ Percentage of Isolates inhibited (\%) } \\
\hline & & Zn & Azy & Au & Avf & Sf & $\mathbf{L V}$ & Gax & Cf & $\mathbf{S}$ & Apx & $\mathbf{C N}$ & $\mathbf{N}$ \\
\hline Staph. Aureus & 32 & 93.8 & 93.8 & 9.4 & 84.4 & 75 & 100 & 53.1 & 100 & 56.3 & 3.1 & 40.1 & 0 \\
\hline Strept. Species & 13 & 92.3 & 100 & 0 & 76.9 & 46.2 & 84.6 & 23.1 & 100 & 84.6 & 0 & 76.9 & 0 \\
\hline Pseudo. aerugin. & 33 & 90.9 & 90.9 & 6.1 & 66.7 & 57.6 & 90.9 & 48.5 & 100 & 81.8 & 0 & 36.4 & 0 \\
\hline Proteus Mirabilis & 16 & 81.3 & 100 & 12.5 & 87.5 & 81.3 & 100 & 68.8 & 100 & 43.8 & 25 & 56.3 & 0 \\
\hline Escheri. Coli & 20 & 95 & 90 & 20 & 80 & 70 & 95 & 45 & 100 & 40 & 20 & 60 & 5 \\
\hline
\end{tabular}


Table 4. Prevalence of Glucouria amongst Patients with Prolonged Healing Orthopaedic Wounds.

\begin{tabular}{lllll}
\hline \multirow{2}{*}{ Duration of Wound (Months) } & \multirow{2}{*}{ Number Examined } & \multirow{2}{*}{ Number with Glycosuria (\%) } & \multicolumn{2}{l}{ Bacterial Infection (\%) } \\
\cline { 4 - 5 } & 3 & $0(0.0)$ & $2(66.7)$ & $1(33.3)$ \\
\hline $1-3$ & 17 & $5(2.9)$ & $17(100.0)$ & $0(0.0)$ \\
$4-6$ & 30 & $17(56.7)$ & $30(100.0)$ & $0(0.0)$ \\
$7-9$ & 50 & $31(62.0)$ & $50(100.0)$ & $0(0.0)$ \\
$10-12$ & 1 & $1(100)$ & $0(0.0)$ & $1(100)$ \\
$>12$ & 101 & $54(53.5)$ & $99(98.2)$ & $2(1.8)$ \\
Total & & & \\
\hline
\end{tabular}

$$
\begin{aligned}
\text { Key: } & =\text { Prevalence of Bacteria Infection } \\
& =\text { No Bacteria Infection } \\
& =\text { Prevalence of Glucose in Urine }
\end{aligned}
$$

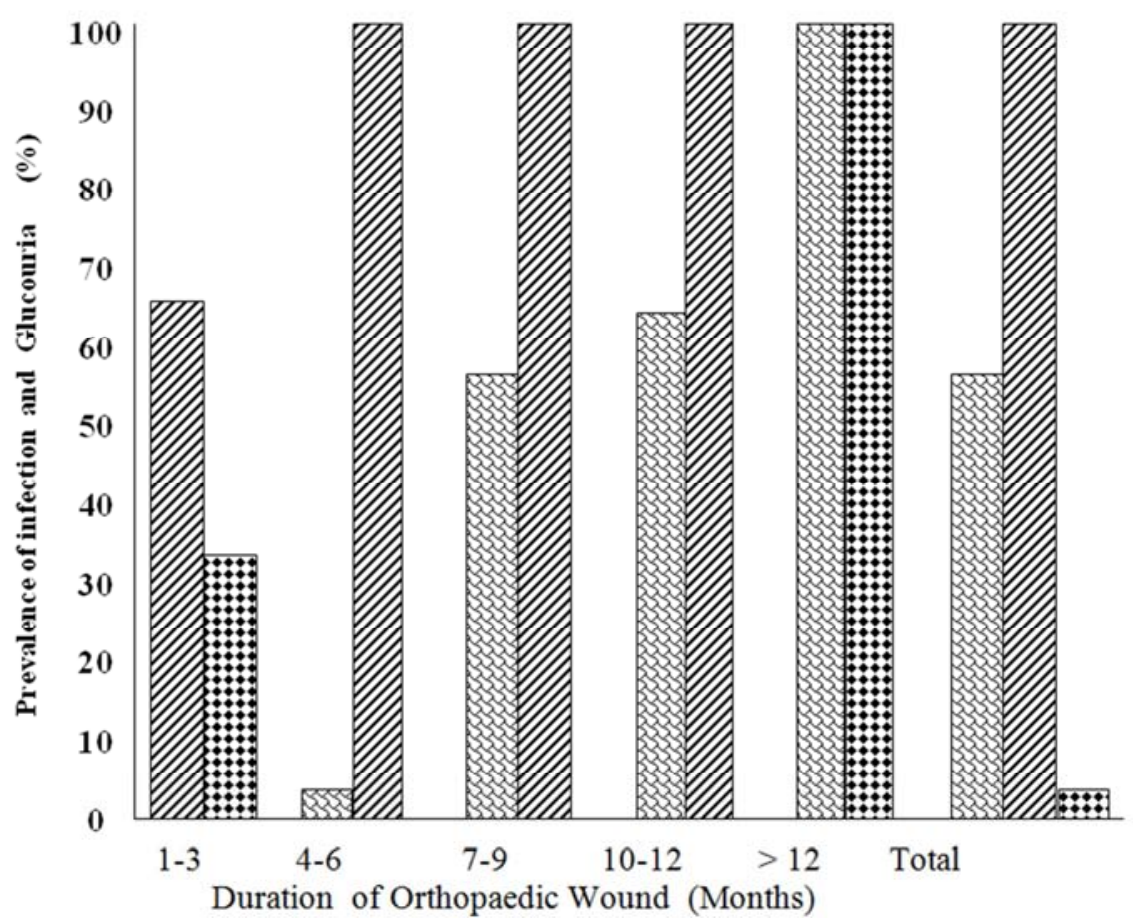

Figure 1. Comparative Analysis of Bacteria infection and Glycosuria in Orthopaedic Wound Patients

\section{Discussion}

Prolonged healing wounds also referred to as non - healing wounds are chronic wounds / injuries that are yet to heal after 6 weeks. This is a growing health challenge in Nigeria and other countries. Previous workers $[11,12]$ reported that nonhealing wounds result in enormous health care expenditures, with the total cost estimated at more than $\$ 3$ billion per year. The present study identified 130 patients with chronic wounds ranging from 3 months to over 12 months, in 6 hospitals out of hundreds of hospitals in Imo State. If all the hospitals and local orthopaedic care centres in the State are covered in the survey, there is no doubt that the population of prolonged - healing orthopaedic wound patients in the State will be running into thousands. Previous workers [13] reported that non-healing wounds affect about 3 to 6 million people in the United States. Elsewhere, the U. S. Centers for Disease Control and Prevention reported that non - healing wounds affect some 5.7 million American adults. Many reasons are responsible for these non-healing wounds. The present study identified 2 major factors that cause prolonged healing of the wounds as microbial infections and diabetes mellitus. This finding agrees with previous reports [14], which listed 3 reasons why many wounds would not heal: poor circulation, fluid buildup and infection. In another report, $[14,15]$ explained that wound healing is more complicated among older adults than younger ones and therefore some wounds in adults would delay in healing due to 3 basic reasons: delayed inflammatory response, diabetes and reduced skin elasticity. According to a 2014 National Diabetes Statistics Report, approximately 11.2 million people over the age 65 have diabetes [14]. This disease can slow down wound healing process for several different reasons. 
Wound Care Centers points out that elevated blood sugar levels in diabetic patients narrows blood vessels and hardens the arteries, both negatively affect wound healing [14]. Diabetic neuropathy, which results in loss of sensation, can also slow down wound healing. In the present study, 53.5\% of patients with prolonged - healing wounds have glycosuria (indicating diabetes).

Micro - organisms are ubiquitous and adapted to different environmental conditions. Many live as normal flora on the skin of healthy persons. Once the skin is injured, microorganisms that are normally sequestered at the skin surface obtain access to the underlying tissues. All patients in this study had one microbial infection or the other, indicating that microbial infection contributed significantly in delaying healing. According to [16], the state of infection and replication status of the micro-organisms determines whether the wound is classified as having contamination, colonization, local infection/critical colonization, and/or spreading invasive infection. Inflammation is a normal part of the wound-healing process, and is important to the removal of contaminating micro-organisms. In the absence of effective decontamination, however, inflammation may be prolonged, since microbial clearance is incomplete. Both bacteria and endotoxins can lead to the prolonged elevation of pro-inflammatory cytokines such as interleukin-1 (IL-1) and TNF- $\alpha$ and elongate the inflammatory phase. If this continues, the wound may enter a chronic state and fail to heal. This prolonged inflammation also leads to an increased level of matrix metalloproteases (MMPs), a family of proteases that can degrade the ECM. In tandem with the increased protease content, a decreased level of the naturally occurring protease inhibitors occurs. This shift in protease balance can cause growth factors that appears in chronic wounds to be rapidly degraded $[12,16]$. Similar to other infective processes, the bacteria in infected wounds occur in the form of biofilms, which are complex communities of aggregated bacteria embedded in a self-secreted extracellular polysaccharide matrix [16]. Mature biofilms develop protected microenvironments and are more resistant to conventional antibiotic treatment. The microbes implicated in the present study: Staphylococcus aureus, Pseudomonas aeruginosa, Eschericia coli, Proteus mirabilis and $\beta$ hemolytic streptococci are common bacteria in infected and clinically non-infected wounds as also observed by previous workers [16, 17]. Elsewhere, previous workers [15] reported that Psedomonas aeruginosa and Staphylococcus appear to play an important role in bacterial infection in wounds. Many chronic ulcers probably do not heal because of the presence of biofilms containing Pseudomonas. aeruginosa, thus shielding the bacteria from the phagocytic activity of invading polymorphonuclear neutrophils. This mechanism may explain the failure of antibiotics as a remedy for chronic wounds.

\section{Conclusion}

The present study did not carry diagnostic tests on the HIV status of the patients, however $8.9 \%$ of them indicated in their questionnaires that they are known HIV positive patients. HIV infection reduces CD4+ cells which play major role in conferring immunity at the presence of infection. Earlier studies $[18,19]$ suggest that delayed T-cell infiltration along with decreased T-cell concentration in the wound site is associated with impaired wound healing, while others have reported that CD4+ cells ( $\mathrm{T}$-helper cells) have a positive role in wound healing and CD8+ cells (T-suppressor-cytotoxic cells) play an inhibitory role in wound healing. Interestingly, recent studies in mice deficient in both $\mathrm{T}$ - and $\mathrm{B}$-cells have shown that scar formation is diminished in the absence of lymphocytes [20]. In the light of these findings, we conclude that microbial infections and glycosuria are major factors contributing to prolonged healing orthopaedic wounds in Imo State, south eastern Nigeria. We recommend that orthopaedic wound patients be screened for HIV infection and those found positive should be placed on anti retroviral drug therapy in addition to their wound management therapy.

\section{Acknowledgements}

The authors are grateful and appreciate the assistance of all medical doctors, nurses and medical laboratory scientists who assisted in one way or the other to make this study a success. We particularly thank Rachael Oparaji and Chinelo Chukwudiaru of Imo State University Teaching Hospital Orlu and Mrs. Chidinma Irehie of Federal Medical Centre Owerri amongst others

\section{References}

[1] Harvey, Carol (2005): Wound Healing. Orthopaedic Nursing, National Association of Orthopaedic Nurses 24 (2) 143 - 157.

[2] Bishop A. (2008). Role of oxygen in wound healing. J Wound Care 17: 399-402.

[3] Rodriguez PG, Felix FN, Woodley DT, Shim EK. (2008). The role of oxygen in wound healing: a review of the literature. Dermatol Surg 34: 1159-1169.

[4] Guo, S. and DiPietro, L. A. (2010): Factors Affecting Wound Healing. Journal of Dental Research. 89 (3): 210 - 229.

[5] Han, B. R., Oh YS, Ahn, K. H., Kim H. Y., Hong S. C., Oh M. J., Kim H. J., Kim Y. T., Lee K. W., Kim S. H., B. R., Han (2010):. "Clinical Implication of 2nd Trimester Glycosuria." Korean Journal of Perinatol. 21 (3): 258-265.

[6] Rose, Burton; Rennke, Helmut (1994). Renal pathophysiology - the essentials (1st ed.). Philadelphia: Lippincott Williams \& Wilkins. p. 194. ISBN 0-683-07354-0.

[7] Obiajuru I. O, C. and Ozumba, U. C. (2017): Laboratory Methods for Medical Microbiology \& Parasitology. Revised Edition. Lifeway Amalgamations. Owerri. ISBN: 25799107. p. 168.

[8] Chesbrough, M. (2002): Medical Laboratory Manual for Tropical countries vol II: Microbiology. Tropical health Technology/Butterworths and Co Ltd. Cambridge/Sevanaks. 
[9] Holt, J. G., Krieg, N. R., Sneath, P. H. A., Stanley, J. T. and Williams, S. T. (1998): Bergy's manual of determinative bacteriology. Baltimore, Md: Williams and Wilkins Publishing Co. Maryland.

[10] Philip, L. O. (1973): Bayelsian Statistics for social scientists. Whitefriar Press. London p 215.

[11] RCH Orthopaedic (2010): Orthopaedic wound care. RCH Orthopaediac Department. Internet.

[12] Menke NB, Ward KR, Witten TM, Bonchev DG, Diegelmann RF. (2007). Impaired wound healing. Clin Dermatol 25: 1925.

[13] NCBI - NIH (2016) Factors affecting Wound Healing. https:/www.ncbi.nlm.nih.gov/pmc/articles/PMC2903966/

[14] Advanced Tissue (2015): Why Do Elderly People Experience Delayed Wound Healing? https://www.advancedtissue.com/why-do-elderly-peopleexperience-delayed-wound-healing/
[15] Bjarnsholt T, Kirketerp-Moller K, Jensen P, Kit M, Krogfelt $\mathrm{K}$, Phipps R, et al. (2008). Why chronic wounds won't heal: a novel hypothesis. Wound Repair Regen 1: 2-10.

[16] Edwards R, Harding KG. (2004). Bacteria and wound healing. Curr Opin Infect Dis 17: 91-96.

[17] Davis SC, Ricotti C, Cazzaniga A, Welsh E, Eaglstein WH, Mertz PM. (2008). Microscopic and physiologic evidence for biofilm-associated wound colonization in vivo. Wound Repair Regen 16: 23-29.

[18] Swift ME, Burns AL, Gray KL, DiPietro LA. (2001). Agerelated alterations in the inflammatory response to dermal injury. Journal of Invest Dermatol 117: 1027-1035.

[19] Park JE, Barbul A. (2004). Understanding the role of immune regulation in wound healing. Am J Surg 187: 11-16.

[20] Gawronska-Kozak B, Bogacki M, Rim JS, Monroe WT, Manuel JA. (2006). Scarless skin repair in immunodeficient mice. Wound Repair Regen 14: 265-276. 